\title{
Towards a PURE Spoken Dialogue System for Information Access
}

\author{
Rajeev Agarwal \\ Media Technologies Laboratory \\ Texas Instruments Inc. \\ PO Box 655303, MS 8374 Dallas, TX 75265 \\ USA \\ rajeev@csc.ti.com
}

\begin{abstract}
With the rapid explosion of the World Wide Web, it is becoming increasingly possible to easily acquire a wide variety of information such as flight schedules, yellow pages, used car prices, current stock prices, entertainment event schedules, account balances, etc. It would be very useful to have spoken dialogue interfaces for such information access tasks. We identify portability, usability, robustness, and extensibility as the four primary design objectives for such systems. In other words, the objective is to develop a PURE (Portable, Usable, Robust, Extensible) system. A two-layered dialogue architecture for spoken dialogue systems is presented where the upper layer is domainindependent and the lower layer is domainspecific. We are implementing this architecture in a mixed-initiative system that accesses Hight arrival/departure information from the World Wide Web.
\end{abstract}

\section{Introduction}

With the rapid rate at which the availability of information is increasing, it is important to make access to this information easier. One may wish to get the arrival/departure information for a given flight, verify if a particular book is available at a library, find the stock price for any fund, access yellow page information on-line, check/maintain voice mail remotely, get schedules for entertainment events, perform remote banking transactions, get used car prices, and the list goes on and on. Such tasks can be classified as information access (IA) tasks, where the primary objective is to get some piece of information from a certain place by providing constraints for the search. Some of these tasks may also involve an "action" that may change the state of the underlying database, e.g., making a reservation for an event, making transactions on an account, etc. It would be very helpful to develop Spoken Dialogue (SD) interfaces for such IA applications, and several such attempts are already being made (Seneff et al., 1996; Sadek et al., 1996; Abella et al., 1996; Fraser and Dalsgaard, 1996; Lamel et al., 1996; Kellner et al., 1996; Niedermair, 1996; Barnett and Singh, 1996; Gorin et al., 1996).

In this paper, we differentiate between such IA tasks and the more complicated problem solving tasks where multiple sub-problems are concurrently active, each with different constraints on them and the final solution consists of identifying and meeting the user's goals while satisfying these multiple constraints. Examples of such applications include a system that offers investment advice to a user based on personal preferences and the existing market conditions, or an ATIS-like application that assists the user in travel planning including flight reservations, car rental, hotel accommodations, etc.

In addition to the general requirement of accuracy, there are four other important design objectives for SD systems:

- Portability of an SD system refers to the ability of the system to be moved from one application/domain to another.

- Usability of an SD system refers to the ease with which a user can use the system and the naturalness that it provides.

- Robustness of an SD system refers to the ability of the system to help the user acquire the desired information even in the presence of user and system errors.

- Extensibility of an SD system implies that additional queries within a given application can be added to the system without much trouble. 


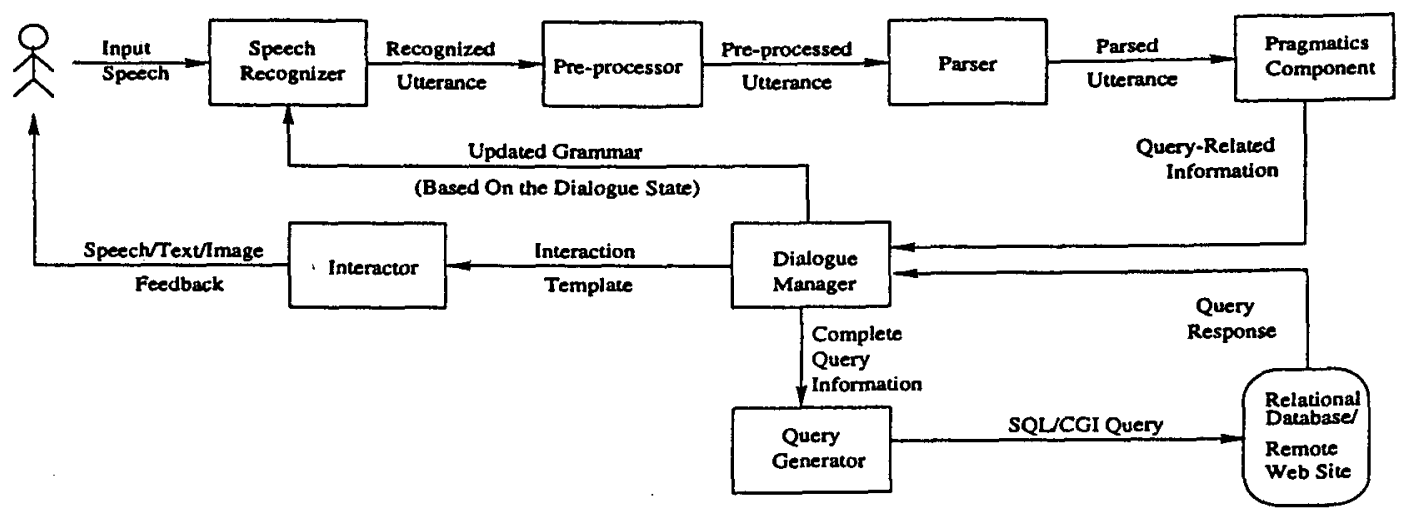

Figure 1: Outline of the Components of the Spoken Dialogue System

The purpose of this paper is to describe an SD system, in particular the dialogue manager, that is being developed with these objectives in mind. Since these design objectives are often conflicting in nature, one has to strike a balance between them. In a manner of speaking, one could say that the objective is to create a PURE (Portable, Usable, Robust, Extensible) system. It is our belief that it is possible to develop an "almost" PURE system for IA tasks.

\section{Overall System Description}

The overall SD system is responsible for taking user utterances as input, processing them in a given context in an attempt to understand the user's query, and satisfying his/her request. The user does not need to know anything about the structure of the database or the architecture of the system. In case the user's utterance has missing, ambiguous, inconsistent, or erroneous information, the system engages the user in a dialogue to resolve these. The system is designed to be mixed-initiative, i.e., either the user or the system can initiate a dialogue or subdialogue at any time. The dialogue ends when the user decides to quit the system. The system can be used for querying a relational database using SQL or invoking a CGI ${ }^{1}$ script on the web. A brief overview of the different components is presented in Figure 1.

- Speech Recognizer: It is responsible for recognizing the user utterance and producing a

${ }^{1}$ CGI stands for Common Gateway Interface. It is a tool that assists web programmers in creating interactive, user-driven applications. Several web sites permit database queries where the user types in the search constraints on an HTML FORM and the server submits this form to the CGI script which generates a response after searching a local database. Note that here we refer to such database searches and not to the string searches as offered by Lycos, WebCrawler, Excite, etc. recognition string. We currently write separate context-free grammars for each state of the dialogue and use these to recognize the utterances with the DAGGER speech recognition system described in (Hemphill and Thrift, 1995). An important feature of this recognizer is that based on the dialogue state, certain grammars may be switched into or out of the dynamic vocabulary ${ }^{2}$, thereby leading to better speech recognition accuracy.

- Preprocessor: This component is responsible for identifying domain-independent (e.g., time, place name, date) and domain-specific semantic patterns (e.g., airport name, book title) in the input utterance.

- Parser: Since user utterances could be ungrammatical in nature, a partial parser has been implemented to parse the input utterance into its component phrases. This provides added robustness, although lack of a deep structure in the parse sometimes causes the pragmatics component to miss useful information.

- Pragmatics Component: This component is responsible for identifying the values of relevant fields that are specified in the utterance, based on the partial parse of the utterance. It uses an application specific input file called the application schema, which describes all the relevant fields in that application and lexico-semantic patterns that indicate their presence. It also describes the possible queries that may be made in that application.

\footnotetext{
${ }^{2}$ We only use the grammar switching feature of DAGGER, but it offers the ability to load completely new grammars dynamically if such a need arises.
} 
- Dialogue Manager: It evaluates the knowledge extracted by the pragmatics component to determine the current state of the dialogue. It processes this new dialogue state and constructs an "interaction template" that determines what feedback should be provided to the user.

- Query Generator: This component is responsible for generating a database query. It can generate either a SQL query for a relational database or a CGI script query for querying a web site.

- Interactor: It is responsible for converting the interaction template generated by the dialogue manager into English sentences that can be printed and/or spoken (using a text-to-speech system) to the user to provide feedback. It uses a template-to-string rules file that contains rules for all possible types of interactions. In some cases, it may also provide feedback by updating a displayed image.

This gives a brief overview of our SD system. The system is still under development, and is being tested on the flight arrival/departure information application for which we query the American Airlines web site (American Airlines, 1997). System development is expected to be completed soon. We have also used this system to begin developing a "Map Finder" demo that queries the MapQuest web site (MapQuest, 1997) to display maps of any street address or intersection in the United States. We intend to port this system to the yellow pages information access application in the near future.

\section{Dialogue Manager Design}

\subsection{Background}

Existing approaches to designing dialogue managers can be broadly classified into three types: graphbased, frame-based, and plan-based. This section gives a brief overview of these approaches and argues that for IA tasks, the frame-based approaches are the most suitable.

Graph-based approaches require the entire dialogue state transition graph for an application to be pre-specified. Several dialogue design toolkits are available to assist developers in this task, such as the SLUrp toolkit (Sutton et al., 1996), SpeechWorks toolkit (Applied Language Technologies, 1997), or DDL-tool (Baekgaard, 1996). It is often cumbersome and sometimes impossible to pre-specify such a dialogue graph. Further, such approaches are not robust as they cannot appropriately handle any unforeseen circumstances.
Plan-based approaches attempt to recognize the intentions of the entities involved in the discourse and interpret future utterances in this light. They are usually based on some underlying discourse model, several of which have been developed over the years (Cohen and Perrault, 1979; Mann and Thompson, 1983; Grosz and Sidner, 1986; Carberry, 1990). We argue here that although plan-based systems are very useful for problem-solving tasks like the ones described earlier, that degree of sophistication is not needed for IA tasks. For example, of the five types of intentions outlined by Grosz and Sidner (1986), only "intent that some agent believe some fact" and "intent that some agent know some property of an object" are encountered in IA tasks, and they can be easily conflated for such tasks, without any loss of information. Further, although modeling a speaker's intentions and the relations between them is informative about the structure of the discourse, their recognition in an actual system may be non-trivial and prone to errors. Most IA tasks have only one discourse purpose, and that is to get some information from the system. The various discourse segments are all directed at providing the system with relevant constraints for the database query. Therefore, explicit modeling of the discourse purpose or discourse segment purpose is unnecessary.

Frame-based systems typically have a domain/application model to which they map user utterances in an attempt to recognize the nature of the user's query. The constraints of the application drive the analysis of utterances. Such systems usually ignore phenomena like diectic references, expressions of surprise, discourse segment shifts, etc.

\subsection{Two-Layered Architecture}

It is our contention that for IA tasks, the dialogue between the user and the system proceeds in a domain-independent manner at a higher level and can be described by a set of domain-independent states. Some domain-specific interactions are required once the dialogue is in one of these higher level states and these can be described by a different set of states. This view of the structure of the dialogue led us to a two-layered architecture for the DM. The upper layer is completely domain-independent, while the lower layer has dialogue states that constitute domain-specific subdialogues. Further, although the different states of the dialogue are pre-specified, the system automatically identifies what state it is in based on the user's utterance, the result of the database query, and knowledge of the previous dialogue state. This is what Fraser and Dalsgaard (1996) refer to as a 


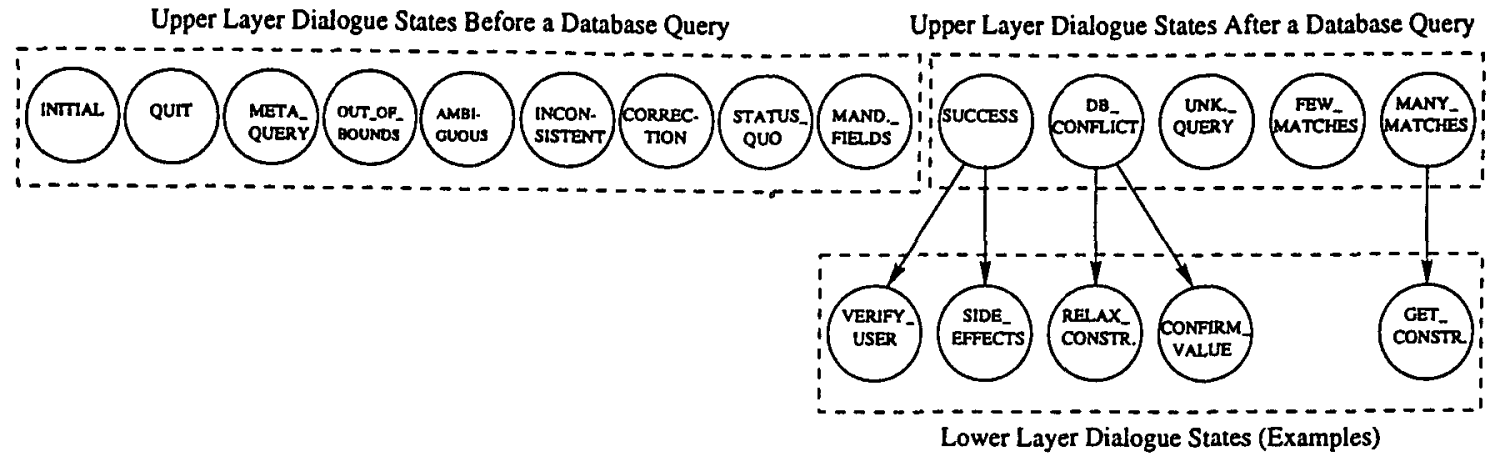

Figure 2: States in the Two-Layered Dialogue Management Architecture

self-organizing system. Most plan-based and framebased systems are self-organizing. The states in the DM are shown in Figure 2 and are described in detail in this section.

\subsubsection{Dialogue States}

All fourteen states presented here at the top level belong to the upper layer of the dialogue. For some of these upper layer states, references are made to the lower layer states that they may spawn to accomplish domain-specific sub-dialogues. After every user utterance, the DM checks to see if the dialogue is in one of the upper layer dialogue states. Lower layer states are checked only if the system is already in a sub-dialogue. The upper layer states are tried in the order in which they are described below since if the dialogue is in any of the earlier states, there is no point in trying later ones. The existence of one of the first nine states listed below may be determined without a database query. If the dialogue is not in any one of these nine states, then there is enough information to issue a query, and the dialogue may be in one of the last five states based on the results of the query. The dialogue ends when the QUIT state is reached.

1. INITIAL: This is the state in which each dialogue starts and reverts to after a query made by the user has been completely processed.

2. QUIT: If the system detects that the user wants to terminate the current dialogue, then the dialogue enters this state.

3. META_QUERY: The dialogue reaches this state when the user either explicitly asks for help (e.g., "Please help me," "what can I say," etc.) or asks for some meta-level information about the system's capabilities (e.g., "what cities do you know about?"). The help messages in the system are context-sensitive and are based on the current dialogue state.

4. OUT_OF_BOUNDS: This state is reached when the system realizes that the user either wants to access information that the system is not equipped to handle or access "legitimate" information in ways the system is not designed to handle. For example, if a system is designed to access American Airlines flight information and the user says "what time does Delta flight 472 reach Dallas?," the system enters the OUT_OF_BOUNDS state. An example of an improper legitimate query could be "what time does my plane leave?," if the system expects the word 'flight' but not 'plane'. The objective is not just to quit gracefully, but to allow the user to re-enter the dialogue at some place. In the first case, the system informs the user of the limitations of the system, switches the dialogue to the INITIAL state, and permits the user to revert to some query within the bounds of the system. In the second case, it informs the user that the word 'plane' is unknown to the system, and requests him/her to rephrase the query.

5. STATUS_QUO: This state is reached if the system determines that the most recent utterance by the user provided no additional query-related information to the system. This is an indication that the user was either completely silent, did not know the answer to the system's previous question (may have responded by saying "I don't know" to something the system had asked), explicitly asked the system to repeat the last feedback (may have said "Can you repeat that"), the speech recognizer misrecognized the part of the utterance that was meant to be informational, or the utterance really had no new 
information. Based on what the user said, an appropriate response is generated.

6. AMBIGUOUS: This state is reached when one of three types of ambiguities exists in the system. Lexical ambiguity arises if some user term matches two entities within the same semantic class. For example, in a library application, if the user asks for "Dickens" and the database contains two or more authors with that last name, this term is lexically ambiguous. Class ambiguity arises if a term may belong to two or more semantic classes. In the above example, if there is also a book entitled "Dickens" in the database, then class ambiguity exists since it is unknown whether the user meant the 'author' or the 'title'. This can often be resolved based on the surrounding context. Field ambiguity arises when the system has found a term that could refer to more than one database field. For example, in a flight arrival/departure application, if the system prompts the user for either the arrival city or departure city, and the user just says "Newark," the field to which the term belongs is ambiguous.

7. INCONSISTENT: User or system errors may sometimes lead the DM to this state where the system's knowledge of the various fields violates some consistency rule. The consistency rules specific to an application are provided in an input file. For example, an error may cause the system to believe that the departure city and the arrival city in a flights arrival/departure application are the same. If that happens, the user is notified of the inconsistency so that the error may be rectified.

8. CORRECTION: This state is reached when the system realizes that the user is attempting to correct either an error the user may have made or an error made by the recognizer. As a result, the system accepts the corrected value provided by the user (assuming that this new value is correctly recognized) and provides appropriate feedback. For example, in a flight arrival/departure application, the user might say "I said Dallas, not Dulles" to correct a misrecognition by the speech recognizer.

9. MANDATORY_FIELDS: This state is needed only for applications in which values for certain fields must be known before a query can be issued. This is often true of applications that invoke CGI scripts on the web. For example, the American Airlines web site only permits a query if the user specifies either the flight number, or the arrival and departure city and approximate arrival time, or the arrival and departure city and approximate departure time. This state ensures that values for these mandatory fields are obtained from the user before issuing a CGI query.

10. SUCCESS: If none of the previous states were found, a query is issued to the system. If this query results in a successful match, then the dialogue is in this state. After providing appropriate feedback to the user, the system performs a further check to see if any "action" needs to be carried out on the accessed item(s) of information. For example, in a banking application, having checked the balance in a savings account, the user may now wish to transfer money from checking to savings. This state usually spawns a sub-dialogue which may or may not be domainspecific. The lower level dialogue states in this sub-dialogue could be -

- VERIFY_USER: which asks for the user's account ID and password,

- SIDE_EFFECTS: which informs the user of some side effects of the imposed constraints, e.g. "This transaction will lead to a negative balance in the checking account," or

- some other domain-specific state depending upon the nature of the action involved.

Once in this state, the user may start a new query, ask for more information about the matched item, or quit the system.

11. DATABASE_CONFLICT: A database conflict arises when the constraints specified by the user do not match any item in the database. This could be because of conflicting information from the user or speech recognition errors. Such conflicts must be resolved before proceeding in the dialogue. Conflict resolution may be accomplished by a sub-dialogue in the lower layer. Some of the possible states in the lower layer are:

- RELAX_CONSTRAINT: asks the user to relax a certain constraint, e.g., "No Thai restaurant found on Legacy, but there is one on Spring Creek - is that OK?" (the system needs domain-specific information that Legacy and Spring Creek are close to each other). In some cases, the system also 
needs to know which constraints are "negotiable".

- CONFIRM_VALUE: asks the user to confirm some field values provided by the user. The confirmation is needed to ensure that it was not a system or user error that caused a conflict.

12. UNKNOWN_QUERY: In most applications, the user may query for different types of information. In a yellow pages application, for example, the user may ask about a phone number, an email address, or a postal address. The DM may need to know what item of information the user is interested in, as this determines the feedback provided to the user. This is especially useful in applications without a display (queries made over the telephone) since it takes time to give more information than is necessary. Note that it is often possible to issue a database query even if this information is not known, and that is why this state belongs to the set of possible states after a query has been made.

13. FEW_MATCHES: If the database query results in a "few" matches, then the dialogue enters this state. Whenever few matches are found, the most efficient way to consummate the query is to enumerate these matches so the user can the select the one of interest.

14. MANY_MATCHES: If none of the previous states are reached, the database query must have resulted in too many matches, i.e., not enough information was supplied by the user to match only a single or a few database items. This state may spawn a domain-specific subdialogue in the lower layer, one of whose states could be:

- GET_CONSTRAINT: The objective is to ask the user to specify the least number of constraints that lead to the SUCCESS state. So, whenever possible, this dialogue state identifies what piece of information would be "most informative" at that point in time, and asks the user to specify its value.

This concludes the description of the various dialogue states. While we have attempted to provide an upper layer that covers most IA tasks, the lower layer states given here are just examples of some possible states. Depending upon the application, more lower layer states can be added to improve the usability/robustness of the system.

\section{Comparison to Other Approaches}

Several other mixed-initiative spoken dialogue systems have been developed for information access tasks (Abella et al., 1996; Bennacef et al., 1996; Kellner et al., 1996; Seneff et al., 1996; Fraser and Dalsgaard, 1996; Sadek et al., 1996; Barnett and Singh, 1996) and they provide varying degrees of dialogue management capability. Our dialogue management approach is possibly most similar to that proposed by Abella et al. (1996), with some important differences. We have attempted to clearly define a comprehensive set of states to handle various contingencies including out-of-bounds queries, meta-queries, ambiguities, and inconsistencies due to user/system errors. We feel that our two-layered architecture should make the system more portable. We further contend that if one encounters a dialogue state that is not covered by our state set, it can be abstracted to an upper level state which may later be useful in other applications. Abella et al. (1996) do present a nice question selection methodology that we lack $^{3}$. We currently resort to a domaindependent GET_CONSTRAINT state but hope to improve on that in the future.

The primary bottleneck in our system at this time is the parser which only identifies partial parses and does not perform appropriate PP-attachment, conjunct identification, or do anaphora resolution or ellipsis handling. We need to replace the existing partial parser with a better parser to improve the overall system accuracy.

\section{How PURE is it?}

We started out by saying that the objective is to develop a PURE spoken dialogue system for information access tasks. We want to ensure that our system aims to be as PURE as it can be. In this section, we list those features of our system that are intended to make it PURE.

- Portability:

- In order to move the SD system to a new domain, the following files must be specified: an application schema that was briefly described in Section 2; a schemato-database mapping file that maps items in the application schema to the fields in the relational database or in the CGI script (e.g., the flight_number schema

\footnotetext{
${ }^{3}$ It may be noted that such a methodology is possible only with local relational databases. It cannot be implemented when querying CGI scripts on the web since we do not have access to the underlying database.
} 
field maps to the fltwumber field in the CGI script); a user-to-database mapping file that consists of the various ways a user may refer to a value of a database field (e.g., "Big Apple" maps to "New York"); and a consistency-rules file.

- The two-layered architecture ensures that the overall dialogue progresses at a domainindependent level, and keeps the domainindependent and domain-specific states separate.

- Self-organizing dialogue structure makes it more portable.

- Partial parser can be directly ported to a new domain.

- Usability:

- Mixed-initiative approach helps to promote usability.

- Feedback provided by the interactor can be made more domain-friendly by specifying some extra domain-specific rules at the top of the template-to-string rules file, since these rules are executed in the order specified.

- User may say "I don't know," "Please help me," "What can I say," etc. at any time to get some guidance. The help messages are context-sensitive.

- We intend to add prompt randomization, as suggested by Kellner et al. (1996) to make the interactions "less boring."

- The OUT_OF_BOUNDS state and the META_QUERY state improve usability by informing the user of why a certain utterance was inappropriate and allowing the user to ask about the system's abilities respectively.

- Robustness:

- Partial parser can handle ungrammatical input.

- Lexico-semantic pattern matching for field values ensures that misrecognition of a part of the utterance will still extract useful information from the correctly recognized part.

- The CORRECTION and INCONSISTENT states increase the robustness of the system by making it possible to continue even in the presence of errors.

- Extensibility:
- Additional queries can be added to any application by specifying the query semantics in the application schema and any new fields that they may need.

\section{Final Comments}

We have presented a dialogue management architecture that is mixed-initiative, self-organizing, and has a two-layered state set whose upper layer is portable to other applications. The system is designed to generate either SQL queries or CGI script queries, which makes it capable of querying the vast amount of information available on the World Wide Web.

Although the generation of CGI queries is driven by the schema-to-database and user-to-database mappings files, some degree of application specific work still needs to be performed. One has to experiment with the web site and study the source pages for the HTML FORMS screens in order to create these mappings files and possibly write additional code to generate the appropriate query. For example, the American Airlines web site provides three different web pages to support queries about flight arrival/departure information. An examination of all three source pages revealed that a hidden field fltAns gets one of three values based on which page invokes the script. A special hack had to be built into the query generator to assign an appropriate value to this field. Generation of proper user feedback requires us to also examine the source page of the result of the query. The main limitation of querying CGI scripts is that if the web site being queried is modified by its creators, slight modifications will have to be made to the query generator to accommodate those changes.

Our initial experience with this system, especially porting it from the flights arrival/departure application to the Map Finder application, has been very encouraging. Map Finder is a simpler task and some of the upper layer states (UNKNOWN_QUERY, FEW_MATCHES, and MANY_MATCHES) never occur in this application. An additional lower layer state called MAP_COMMANDS had to be implemented under the SUCCESS state to allow the user to scroll the displayed map in any direction using spoken commands. This required understanding the way the MapQuest web site handles these map navigation commands. The rest of the DM was easily ported to this new application.

This system is still work-in-progress and more work remains. We intend to continue improving the existing components while also porting the system to other applications so that we can learn from our porting experiences. 


\section{Acknowledgements}

The author wishes to thank Jack Godfrey for several useful discussions and his comments on an earlier draft of this paper; Charles Hemphill for his comments and for developing and providing the DAGGER speech recognizer; and the anonymous reviewers for their valuable suggestions that helped improve the final version of this paper.

\section{References}

Alicia Abella, Michael K. Brown, and Bruce Buntschuh. 1996. Development principles for dialog-based interfaces. In Dialogue Processing in Spoken Language Systems Workshop Notes, pages 1-7, Budapest, Hungary, August.

American Airlines. 1997. Gates and times information request: http://www.amrcorp.com.

Applied Language Technologies.

1997. http://www.altech.com/products.htm.

Anders Baekgaard. 1996. Dialogue management in a generic dialogue system. In Proceedings of the Eleventh Workshop on Language Technology: Dialogue Management in Natural Language Systems, pages 123-132, Enschede. University of Twente.

Jim Barnett and Mona Singh. 1996. Architectural issues in spoken natural language dialogue systems. In Dialogue Processing in Spoken Language Systems Workshop Notes, pages 13-20, Budapest, Hungary, August.

S. K. Bennacef, L. Devillers, S. Rosset, and L. F. Lamel. 1996. Dialog in the RAILTEL telephonebased system. In Proceedings of the Fourth International Conference on Spoken Language Processing, volume 1, pages 550-553, October.

Sandra Carberry. 1990. Plan Recognition in Natural Language Dialogue. MIT Press, Cambridge, MA.

Philip R. Cohen and Raymond C. Perrault. 1979. Elements of a plan-based theory of speech acts. Cognitive Science, 3:177-212.

Norman M. Fraser and Paul Dalsgaard. 1996. Spoken dialogue systems: A European perspective. In Hiroya Fujisaki, editor, Proceedings of International Symposium on Spoken Dialogue, pages 2536, Philadelphia, PA, October. Acoustical Society of Japan.

A. L. Gorin, B. A. Parker, R. M. Sachs, and J. G. Wilpon. 1996. How may I help you? In Proceedings of the IEEE Third Workshop.on Interactive Voice Technology for Telecommunications Applications, pages 57-60. IEEE Communications Society.
Barbara Grosz and Candace Sidner. 1986. Attention, intentions, and structure of discourse. Computational Linguistics, 12(3):175-204.

Charles Hemphill and Philip Thrift. 1995. Surfing the web by voice. In Proceedings of ACM Multimedia, pages 215-222, San Francisco, CA, November 7-9.

A. Kellner, B. Rueber, and F. Seide. 1996. A voicecontrolled automatic telephone switchboard and directory information system. In Proceedings of the IEEE Third Workshop on Interactive Voice Technology for Telecommunications Applications, pages 117-120. IEEE Communication Society.

L. F. Lamel, J. L. Gauvain, S. K. Bennacef, L. Devillers, S. Foukia, J. J. Gangolf, and S. Rosset. 1996. Field trials of a telephone service for rail travel information. In Proceedings of the IEEE Third Workshop on Interactive Voice Technology for Telecommunications Applications, pages 111116. IEEE Communication Society.

W. C. Mann and S. A. Thompson. 1983. Relational propositions in discourse. Technical Report RR83-115, Information Sciences Institute, Marina del Rey, CA.

MapQuest. 1997. Interactive atlas: http://www.mapquest.com.

Gerhard T. Niedermair. 1996. A flexible callserver architecture for multi-media and speech dialog systems. In Proceedings of the IEEE Third Workshop on Interactive Voice Technology for Telecommunications Applications, pages 29-32. IEEE Communication Society.

M. D. Sadek, A. Ferrieux, A. Cazannet, P. Bretier, F. Panaget, and J. Simonin. 1996. Effective human-computer cooperative spoken dialogue: The AGS demonstrator. In Hiroya Fujisaki, editor, Proceedings of International Symposium on Spoken Dialogue, pages 169-172, Philadelphia, PA, October. Acoustical Society of Japan.

Stephanie Seneff, David Goddeau, Christine Pao, and Joe Polifroni. 1996. Multimodal discourse modelling in a multi-user multi-domain environment. In Hiroya Fujisaki, editor, Proceedings of International Symposium on Spoken Dialogue, pages 105-108, Philadelphia, PA, October. Acoustical Society of Japan.

Stephen Sutton, David Novick, Ronald Cole, Pieter Vermeulen, Jacques deVilliers, Johan Schalkwyk, and Mark Fanty. 1996. Building 10,000 spoken dialogue systems. In Proceedings of the Fourth International Conference on Spoken Language Processing, volume 2, pages 709-712, October. 